2. Es sind die Erhitzungsproben mit Schwefelsäure für sechs Ole bei verschiedenen Mengenverhältnissen und bei verschiedenen Ausgangstemperaturen bestimmt worden.

3. Die $\mathrm{M}$ a u mené schen Proben von Ölgemischen und die Abweichungen von den theoretischen Werten, die sich dabei ergeben, sind be. stimmt worden.

4. Mit Hilfe der Säurezahlen und der Erwärmungsproben beim Vermischen zweier Öle miteinander ist eine Erklärung der beobachteten $\mathrm{Ab}$ weichungen versucht worden.

5. Es ist der Einfluß des Lichtes, der Temperatur, des Jodïberschusses und der Versuchsdauer bei der Jodzahlbestimmung mit W a l I e r scher und $\mathrm{W}$ i j s scher Jodlösung bestimmt worden.

6. Die vorgenommenen Jodzahlbestimmungen an Ölgemischen haben gezeigt, daß die gefundenen Werte den theoretischen gegenüber im allgemeinen zu hoch sind.

7. Versuch einer Erklärung dieser Beobachtungen auf Grund von sechs gleichzeitig und verschieden lang dauernden Jodzahlbestimmungen.

8. Es sind Versuche mit Glycerin, Triolein und Ölsäure gemacht worden.

9. Bemerkung über eine Beobachtung der Krystalle von ölsaurem Kupfer.

\section{Das Recht der Angestellten an den Erfindungen.}

\section{Von L. Max Wohlgemuth, Essen (Ruhr).}

In Nr. 26 dieser Zeitschrift1) habe ich, anknüpfend an einen Artikel des Herrn Rechtsanwalts $\mathrm{J}$ a cobson ${ }^{2}$ ), auf einige Punkte aufmerksam gemacht, die meiner Ansicht und Erfahrung nach bei der Beurteilung der Frage nach den Rechten der Angestellten an den von ihnen oder durch sie gemachten Erfindungen meist gar nicht oder doch nicht genügend berücksichtigt werden. Auf Grund dieser Ausführungen sind mir von mehreren Fachgenossen Zuschriften zugegangen, die mir teilweise rückhaltlos zustimmten, teilweise verschiedene Einwendungen machten. Aus allem glaubte ich ersehen zu können, wie notwendig es ist, diese ganze Frage einmal eingehend, und zwar sine ira et studio zu erörtern. Es wird ja leider immer der Fehler gemacht, daß man bei derartigen Streitfragen Partei ergreift und mit mehr oder minder großer Leidenschaft und Schärfe kämpft. Zu einer r i c h t i g e $n$ Erkenntnis kommt man aber doch nur dadurch, daß man ruhig und kühl prüft, wie die Verhältnisse $w \mathrm{i} \mathrm{rkli} \mathrm{ch}$ liegen. Mit Schlagwörtern läßt sich gar leicht kämpfen, man begeistert die Menge damit, ohne sie überzeugen zu müssen; wem aber die Sache selbst am Herzen liegt, der wird tiefer in sie einzudringen verlangen, der wird sich nicht durch Schlagwörter leiten lassen.

Bei dem Streit, der sich seit einiger Zeit über die vorliegende Frage erhoben hat, glaube ich, eine gewisse Verstimmung oder Verbitterung wahr-

1) Diese Z. 20, 1099-1101 (1907).

2) Diese Z. 20, 888-890 (1907). nehmen zu können, die mir $\mathrm{k}$ ü $\mathrm{n}$ s $\mathrm{t} l \mathrm{i}$ c h erzeugt und genährt zu werden scheint. Denn — das wollen wir uns nicht verhehlen, sondern von vornherein hier festlegen - die Frage se 1 bst besteht beinahe schonsolange, wie es Patentgesetze gibt, ohne daß man bisher einseitige, übertriebene Forderungen gestellt hat, ohne daß eine derartige Verbitterung in das Angestelltenverhältnis hineingetragen worden ist, wie es in letzter Zeit geschehen ist. In den Motiven zu dem Entwurfe des deutschen Patentgesetzes vom 25./5. 1877 finden sich zu $\$ 3$ folgende Ausführungen :

„Der Entwurf verleiht dem ersten Anmelder, nicht dem Erfinder, Anspruch auf das Patent. Dieser Grundsatz.... bewirkt, daß der Erfinder an einer möglichst baldigen Anmeldung seiner Erfindung ein Interesse hat, und trägt somit dazu bei, die Geheimhaltung der Erfindungen zu beschränken und deren allgemeine Benutzung zu fördern. Abgesehen von der praktisehen Seite kommen für die Sache aber auch innere Gründe in Betracht. Erfindungen haben, gegenüber der Herstellung neuer Schrift- oder Kunstwerke, never Muster oder Modelle, das Eigentümliche, daß sie nicht eben selten von verschiedenen Personen unabhängig voneinander gemacht werden, daß ferner das Verdienst und der Besitz der Er. findungen nicht immer bei demjenigen ruht, welcher die Tatsache der Erfindung feststellt, sondern bei demjenigen, welcher den allgemeinen, ihr zugrunde liegenden Gedanken und die Mittel zu seiner Durchführung hergegeben hat, daß endlich der Ursprung einer Erfindung, die auf einer Anzahl geistiger, oft von verschiedenen Personen herrübrender Kombinationen beruht, im Streitfalle kaum jemals mit voller Sicherheit auf eine bestimmte Person zurückgeführt werden kann. Die Erfindungen, welche in den Werkstätten eines größeren, mit den Einzelheiten der Versuche nicht selbst befaßten Unternehmers, oder in denjenigen einer Gesellschaft ihren Ursprung haben, bieten in diesen Beziehungen schlagende Beispiele dar. Unter solchen Verhältnissen ist es für das praktische Recht unmöglich, bei der Verleihung des Patentes den Nachweis der geistigen Urheberschaft zu verlangen."

Aus vorstehendem geht hervor, daß man schon beim Entwurfe des ersten deutschen Patentgesetzes die Frage, die uns hier beschäftigt, in Frwägung gezogen hat; die in den oben mitgeteilten Motiven niedergelegte Ansicht wurde denn auch bei den drei Lesungen des Gesetzes in keiner Weise bekämpft. Unsere industriellen Verhältnisse sind nun, soweit ich sie beurteilen kann, heute noch viel mehr als vor 30 Jahren dazu angetan, daß man obige Grundsätze gelten lassen muB. Ich will im folgenden versuchen, dies auseinanderzusetzen, indem ich mich dabei an eine unlängst veröffentlichte Kundgebung $W$. von Si e men $\mathrm{s}^{\text {' }}$ halte $^{3}$ ), die in überaus klarer Weise alle hier in Betracht kommenden Verhältnisse beleuchtet, und die zu lesen ich allen Fachgenossen angelegentlich empfehle.

S i e $m$ e $n$ s stellt im Anfange seiner Ausfüh-

3) Gew. Rechtsschutz u. Urheberrecht 12, 203 bis 213 (1907). 
rungen fest, daß es überwiegend theoretische Erwägungen über die Freiheit der Persönlichkeit, über die Natur des Angestelltenverhältnisses, sowie über das Wesen der Erfindungstätigkeit sind, welche zur Aufrollung der vorliegenden Frage geführt haben, wie dies auch auf anderen Gebieten, die von der sozialen Frage berührt werden, zu beobachten ist. Viel weniger trifft man auf Erwägungen, welche dahin gehen, daß die Lage der industriellen Angestellten ungünstig ist, daß ihnen die Möglichkeit, sich zu entwickeln und vorwärts zu kommen, fehle, und $\mathrm{daB}$ im besonderen die Behandlung der Erfindungsfrage dabei wesentlich mitwirke. Es ist selbstverständlich, daß bei der Mannigfaltigkeit unserer industriellen Verhältnisse die Lage der Angestellten eine verschiedene ist; aber im allgemeinen muß doch anerkannt werden, daß kaum eine andere Bevölkerungsklasse so mannigfache und gute Gelegenheiten, vorwärts zu kommen hat, wie derjenige Teil der technischen Angestellten, der sich durch Fleiß, Kenntnisse, Tüchtigkeit und Erfindungsgabe auszeichnet. Es handelt sich also für diese Angestellten, die naturgemäß bei der Frage des Erfinderrechtes am meisten in Betracht kommen, nicht darum, daß sie für tüchtige Leistungen nicht genügend belohnt werden, sondern darum, ob der Angestellte einen gesetzmä Bigen Anspruch erhalten soll an dem Eigentum der von ihm ausgehenden Patente, ob es auf Grund des allgemeinen Rechtsgefühls notwendig ist, daB ihm dieser Anspruch zugesprochen wird, oder ob es wenigstens vom Standpunkte des Allgemeinwohls nützlich ist, daß in diesem Sinne verfahren wird.

Geht man auf die Natur des Angestelltenverhältnisses näher ein, so kommt man bei der Frage, wie weit dieses Verhältnis reicht, zu der Unterfrage, ob und wie weit der Angestellte berechtigt ist, gewisse Vorbehalte zu machen, gewisse Arten von Tätigkeiten und Tätigkeitsergebnissen von der Wirkung des Angestelltenverhältnisses auszuschließen. Im besonderen wirft sich die Frage auf, ob der Angestellte berechtigt ist, seine Erfindertätigkeit als außerhalb des Angestelltenverhältnisses stehend anzusehen. Derjenige, der bei dem heutigen scharfen Wettbewerbe in seinem Berufe vorwärts kommen will, muB seine g a $\mathrm{nz}$ e $\mathbf{P}$ ers ö $\mathrm{nli}$ c h keit für seinen Beruf einsetzen; auch die Firma, die ihn anstellt, setzt voraus, daß er seine ganzen Kräfte auf die ihm vorgeschriebene Tätigkeit verwendet. Als höchst bedenklich muß die Auffassung bezeichnet werden, daß eine Firma nur Anspruch habe auf die mittleren und durchschnittlichen Leistungen ihrer Angestellten, daß 3 über alles, was darüber hinaus ginge, der Angestellte berechtigt wäre, selbständig und für sich allein zu verfügen. Als solche Uberleistungen werden durchweg die Erfin d ungen angesehen, mögen sie nun bedeutend oder unbedeutend sein, und man kann sohließlich zu der Annahme kommen, daß jemand, der eine Erfindung gemacht hat, unter allen Umständen etwas Besonderes, außerhalb aller Regeln Stehendes, vollbracht hat. Es wird jedoch dabei übersehen, daß in jedem denkenden Menschen etwas von einem Erfinder steckt, und daß auch der durchschnittlich begabte 'Techniker ein gewisses Maß von Erfindergabe besitzen muß. Es würde z. B. nicht möglich sein, die Angestellten einer Firma in zwei Gruppen zu teilen und zu sagen: Dies sind Erfinder, und dies sind keine Erfinder. Auf a llen Gebieten besteht Gelegenheit und Notwendigkeit, neben der Routinearbeit auch Neuarbeit zu leisten und neu sich bietenden Aufgaben in selbständigem Denken und mit handelndem Geiste gegenüberzutreten. Auch auf dem nicht rein technischen Gebiete einer Firma liegt die gleiche Gelegenheit vor für produktive Gedankenarbeit, für Geistesblitze, besondere Eingebungen, für Kunstgriffe, neue Wendungen, originelle Ideen u. dgl. Für das Wesen solcher Geistesprodukte ist es offenbar gleichgültig, auf welchem besonderen Gebiete sie zur AuBerung gelangen, und solche Erzeugnisse des Geistes stehen nicht höher im Range, wenn sie die Bezeichnung einer technischen Erfindung erhalten können.

Es kann nun jetzt die Einschränkung gemacht werden, dal3 es im Grunde auch nicht die Erfindungen sind, für welche das Eigentumsrecht für die Angestellten beansprucht werde, sondern es handle sich lediglich um die $\mathrm{P}$ atente, d. h. um Werte, für die der Staat eine Schutzpflicht ïbernimmt. Dem ist folgendes entgegenzuhalten: Mit der technischen Erfindung allein ist es in der Regel nicht abgetan; sie muß auch durchgeführt werden, und dies erfordert die Aufwendung von Zeit, Kosten, Kapital und Risiko. Alle diese würden in den meisten Fällen nicht übernommen werden, wenn nicht die Aussicht bestände, schließlich einen entsprechenden Ausgleich zurückzugewinnen. Der Angestellte einer Firma braucht sich meist um die technische Durchführung und gewerbliche Verwertung nicht zu sorgen, er kann sich ganz der Jirfjndertätigkeit widmen. Denn die Verwertung oder besser gesagt, die Aufgabe, die Erfindung fïr die Praxis verwertbar zu machen, liegt bei der Firma, nicht bei jhm. Die Firma hat deshalb für die Ausübung ihres Berufes die Patente nötig und nicht der Angestellte.

Eine im industriellen Leben stehende und an der technischen Entwicklung mitarbeitende Firma kann aber ihren Beruf nicht erfolgreich ausüben, wenn sie daran gehindert wird, sich eine wirksame und arbeitsfähige Organisation $z u$ schaffen. Eine solche Organisation muß sich natürlich mit gründen auf sozialer Gerechtigkeit und auf der Fürsorge für die in ihrem Dienste tätigen Menschen. Aber darüber darf man nicht übersehen, daß es die eigentliche Aufgabe der Firma ist, auf dem von ihr gewählten Arbeitsgebiete etwas Tüchtiges zu leisten. Ihre Leistung muß nicht nur wirtschaftlich erfolgreich sein, sondern soll auch befruchtend wirken auf die Fntwicklung des in ihr Arbeitsbereich fallenden technischen Gebietes und hierdurch auch gleichzeitig dem allgemeinen Interesse dienen. Dieser Hauptsache gegenüber darf nicht zu einseitig und zu ausschlieBlich das Sonderinteresse der in einer Firma vereint tätigen Individuen betont werden. Eine Firma ist kein Konglomerat von Individuen, kein Tummelplatz für einen Kampf um die Beute zwischen Inhabern, Aktionären, Direktoren, Angestellten und Arbeitern. Sie ist vielmehr selbst Individuum von besonderer, eigener Art. Wenn man was doch in einem Lande, dessen Wohlfahrt und Zukunft auf der sorgfältigsten Pflege des gewerblichen Lebens und des technischen Fortschritts beruht, selbstverständlich ist - - die hauptsächlichsten Träger der technischen gewerblichen Arbeit mög- 
lichst leistungsfähig erhalten will, so muß alles vermieden werden, was zu einer Lähmung der tätigen Kräfte führen kann. Von diesem Gesichtspunkte muß daher auch jeder Vorsohlag geprüft werden, der auf dem Wege der Gesetzgebung in die innere Organisation der Industrie eingreifen will, um angeblich eine Besserung der Lage der Angestellten zu erzielen.

Der in eine an dem technischen Fortschritte erfolgreich mitarbeitende Firma neu eintretende Angestellte darf nicht glauben, daß er, selbst wenn er noch so begabt ist, in erster Linie der Gebende ist. Im Gegenteil, er ist in weit höherem Maße der Emr.. fangende: Ihm öffnet sich das Aulgabengebiet der Firma, ihm erschließen sich die Quellen des praktischen Lebens, er empfängt die Leitung, den Gedankenaustausch, die gesammelten Erfahrungen, die Gehilfen, die Aufgaben und die Gelegenheiten zu neuen Anregungen, zur Verwirklichung von Plänen und Entwürfen, sowie zur Erprobung des Geschaffenen im praktischen Leben. Die besten und fähigsten Kräfte können ohne Erfolge und unfruchtbar bleiben, wenn es ihnen nicht gelingt, in eine Stellung, in Verhältnisse zu kommen, in denen ihnen der nötige Spielraum und die anregende Gelegenheit zur Betätigung geboten wird.

Bei den Versuchen, neue Aufgaben zu lösen, d. h. Erfindungen zu machen, führt der Weg sehr häufig durch Fehlschläge und Mißerfolge, welche ein wertvolles, aber gleichzeitig auch kostspieliges und gefährliches Lehrmaterial für die Firma und ihre Angestellten darstellen. Daß solche Versuche mit Mißerfolgen bisweilen recht bedeutende Opfer erheischen, daß für die praktische Ausführung von (auch patentierten) Erfindungen oft viel Geld zwecklos ausgegeben wird, weiß jeder, der mit den Verhältnissen unserer Industrie vertraut ist. Und ebenso wie es begabte Chemiker gibt, die beim Lösen von neuen Aufgaben aus dem Rahmen des Gewöhnlichen heraustreten, ebenso gibt es auch - eine nicht abzuleugnende Tatsache - Angestellte, die sich in Mißerfolgen und Verfehlungen in ganz besonderer Weise betätigen. DaB aber die Erfolge den Angestellten, die Mißerfolge der Firma gehören, von einem solchen Leitsatz wird selbst der eifrigste Verfechter des Eigentumsrechtes der Angestellten uns nicht überzeugen können.

Nehmen wir nun einmal den Fall an, es würde in unser Patentgesetz eine Bestimmung aufgenommen, dahin gehend, daß das Eigentumsrecht an den Patenten den Erfind e r n gehöre. Bekanntlich werden sehr viele Patente genommen auf recht unbedeutende Erfindungen, Patente, die nur den Zweck haben, der Firma auf dem von ihr bearbeiteten Gebiete die Bahn frei zu halten und ihren Weg für andere, für die Konkurrenz zu sperren. Eine Firma kann es aber nicht zu einem freien und gegen Ansprüche Dritter gesicherten Wege bringen, wenn ihre eigenen Angestellten, ihre Oberbeamten nun ihrerseits mit dem Anspruche auftreten, daß ihnen die Patente gehören. Die Firma kann gar nicht beschließen, eine nęue Aufgabe in Angriff zu nehmen und Verbesserungen vorzunehmen, wenn sie nicht übersehen kann, mit welchen fremden Rechten dabei zu rechnen ist; ob es die eigenen Angestellten, oder ob es Dritte sind, von deren Erlaubnis es abhängt, ein Patent benutzen zu können, kommt für die Praxis auf dasselbe heraus. Die Firma würde in ihrer Bewegungsfreiheit, in ihrer Entwicklungs- und Betätigungsfähigkeit in bedenklicher Weise gelähmt werden, wenn sie über die Patente, die in ihrem Hause zu dem Zwecke genommen sind, ihre Freiheit zu gewährleisten, nicht in voller Selbständigkeit verfügen könnte. Ein Verhandeln mit dem Angestellten über die Bedingungen der Benutzung erscheint von vornherein ausgeschlossen, da es erfahrungsgemä $B$ eine der schwierigsten Angelegenheiten ist, mit einem Patentinhaber einig zu werden. Der Erfinder hat in der Regel eine ganz andere Meinung von dem Wert seiner Erfindung als derjenige, der ihre Durchführung übernehmen soll. Daß auch der Wert einer Erfindung ein sehr verschie. dener sein kann, je nach der Art und Weise, dem Geschick and der Energie, in technischer sowohl wie kommerzieller Hinsicht, mit welcher die Firma an die Einführung der Erfindung geht, darauf habe ich schon früher aufmerksam gemacht; ich verweise hier nochmals auf meine früheren Ausführungen, auch darüber, daß der Wert einer Erfindung überhaupt und der Teil des Wertes einer Erfindung im besonderen, der auf das Verdienst des einen oder des anderen Angestellten zurückzuführen ist, meist gar nicht festgestellt werden kann4). Hierin liegt wieder eine Quelle von Mißhelligkeiten zwischen der Firma und ihren Angestellten. Auf eine weitere Quelle von Mißhelligkeiten, die beim Austritte der Angestellten aus der Firma entstehen können, habe ich auch schon (a. a. O. S. 1101) hingewiesen.

Si e mens erwähnt noch eine weitere Gefahr, die ich nur kurz berühren will, da ich sie wohl für naheliegend, aber nicht für so bedenklich halte, wie es $\mathrm{S}$ i e m e n s zu tun scheint. Er sagt etwa folgendes : Abgesehen davon, daß die Firma durch das Recht der Angestellten auf die Patente in ihrer Tätigkeit schwer behindert wird, muB auch nicht a uBer Betracht bleiben, daß durch ein solches Recht das Verhältnis der Angestellten zur. Firma und das Verhältnis der Angestellten untereinander noch weiteren Schaden erleidet. Die Bedeutung und die ganze Auffassung von Zweek und Wert der Patente wird eine ganz andere, wenn letztere nicht lediglich dazu dienen sollen, die Durchführung der Aufgaben der Firma zu erleichtern, sondern den Charakter von Sondervorteilen erhalten, welche die Angestellten sich bei Ausübung ihrer Berufstätigkeit im Dienste der Firma durch Benutzung der ihnen in diesem Zusammenhange gewährten Gelegenheiten zu sichern vermögen. Da entsteht die Gefahr, daß die Patente $\mathrm{S}$ e I b s t z w e c k für die Angestellten werden, und die Versuchung an sie herantritt, auf den Weidegründen der Firma in selbstsüchtiger $\mathrm{Ab}$ sicht zu grasen und den offen vor ihnen liegenden Weg der Firma in planmäBiger Weise zu begleiten, um die vorteilhaften Möglichkeiten, Patente zu nehmen, so früh wie möglich zu erkennen und für sich in Sicherheit $\mathrm{zu}$ bringen. -

Nachdem ich im vorstehenden die Verhältnisse, die bei Beurteilung der vorliegenden Frage berücksichtigt werden müssen, kurz dargelegt habe - ich verweise dabei nochmals ausdrücklich auf die ausführlichen Auseinandersetzungen $W$. v. S i e m ens (a. a. O.) -, komme ich zu dem Ergebnis :

4) Vgl. diese Z. 20, 1100 (1907). 
Unsere Industrie würde durch eine $g$ e s e t $\mathrm{z} l$ i c b e Fest le gu $\mathrm{g}$ irgend welcher Art über das Recht der Angestellten an den Erfindungen zweifellos gehindert, geschädigt werden, und unter dieser Schädigung würde im allgemeinen unsere Volkswirtschaft und im besonderen auch wir angestellten Chemiker zu leiden haben. Unser Patentgesetz ist geschaffen worden mit dem Ziele in erster Linie, zugunsten der Entwickelung der hei $\mathbf{m}$ is $\mathrm{ch}$ en Industrie die praktische Verwertung neuer Erfindungen zu befördern ${ }^{5}$ ). In richtiger Erkenntnis der Sachlage hat man von Bestimmungen, die nur Hindernisse für die Industrie bieten würden, abgesehen (s. oben). Auch jetzt sollte es die Gesetzgebung vermeiden, auf Grund einer nicht einwandfreien Theorie die innere Organisation der Industrie zu schwächen und sie dadurch weniger fähig zu machen, die bisherige so kräftige Vorwärtsbewegung mit gleichem Erfolge fortzusetzen.

Man hat mir eingewendet (und wird es nach obigem vielleicht jetzt wieder tun), daß alle vorstehenden Ausführungen sich nur auf $\mathrm{groBe}$ F a b r k e $\mathrm{n}$ beziehen, in denen viele Angestellte Hand in Hand arbeiten, daß sie aber nicht Geltung hätten für kleine Betriebe, in denen vielleicht e i n Angestellter allein arbeitet, allein erfindet. Ich gebe dies gern zu, räume sogar weiter noch ein, daß auch in recht großen Betrieben Fälle vorkommen können, bei denen alles, Anregung, Ausarbeitung, Ausführung der Erfindung von e in ex Person ausgeht. Aber alle diese Fälle sind nur sehr vereinzelt, sie sind die Ausnahmen, die die Regel bestätigen. Es wird doch im Ernste niemandem einfallen, zu verlangen, daß wegen der verhältnismäßig kleinen $Z a h l$ von Fällen _- sie ist wirklich im Verhältnis verschwindend klein -, auf die sich obige Ausführungen nicht anwenden lassen, gesetzliche Bestimmungen erlassen werden, die große Gebiete unserer Volkswirtschaft hemmen und für weite Kreise Nachteile bringen.

Was ich im vorstehenden bekämpft habe, ist die Forderung einer g e s e $\mathrm{tzl}$ i e h e n Festlegung der Rechte der Angestellten an den Erfindungen. Dies schließt jedoch nicht aus, daß 3 ich die Forderung kräftig unterstütze, daß derjenige Angestellte, der durch eine Erfindung (oder sonstige Neuerung) auf dem Arbeitsgebiete seiner Firma diese in besonderem Maße gefördert hat, über das vereinbarte Gehalt hinaus ein Anrecht auf eine besondere Belohnung hat. Wie aber eine solche zu gewähren ist, sei es durch Tantieme, Beteiligung am Reingewinn, dauernde Gehaltserhöhung oder eine einmalige Vergütung, das soll doch, wie bisher, auch weiterhin besonderer privater Verständigung überlassen bleiben. Hier muß je nach dem besonderen Falle entschieden werden. Jeder Firma, die ihren Weg zielbewußt geht, muß doch daran liegen, tüchtige Angestellte $\mathrm{zu}$ fördern, sich ihre Kräfte weiter zu sichern. Je mehr ihre Mitarbeiter zu angesehenen Lebensstellungen sich emporzuschwingen vermögen und zu berühmten Namen gelangen, desto besser wird es mit dem Fortschritt des Hauses bestellt sein.

Zum Schlusse möchte ich nochmals auf das amerikanische Recht hinweisen und der einen Ent-

5) Vgl. z. B. Berger, Das Patentgesetz v. 25./5. 1877 (Guttentag 1884). scheidung, die ich früher ${ }^{6}$ ) angeführt habe, eine zweite anfügen, die ebenfalls zeigt, daß man auch in Amerika bei der Frage nach dem Rechte an den Erfindungen ähnlichen Grundsätzen folgt, wie sie von mir auseinandergesetzt sind. Es ist eine Entscheidung des Court of Appeals of the District of Columbia in der Streitsache Larkin c. Richardson: ich entnehme den Wortiant einem Referate einer deutschen Zeitschrift.7):

„Eine Person hat einen verbesserten Grundsatz für eine Fabrikation entdeckt und verwendet andere zur Unterstützung bei der Ausführung dieses Grundsatzes. Wenn diese Personen bei dieser Tätigkeit wertvolle Zusätze zu dem vorher gefaßten Plane des Auftraggebers machen, so werden diese Verbesserungen im allgemeinen als Eigentum der Partei, welche den ursprünglichen verbesserten Grundsatz entdeckte, angesehen und können in sein Patent als Teil seiner Erfindung eingeschlossen werden."

\section{Die Bedeutung der Lebensversicherung im Wirtschaftsleben der Gegenwart.}

\author{
(Eingeg. d. 18.17. 1907.)
}

Wie im Haushalt de Einzelnen die Erhaltung und Sicherung der erworbenen Güter nicht minder wichtig ist als der Erwerb selbst; so kommt auch im Wirtschaftsleben der Völker den Institutionen, die den materiellen Nutzen des Nationalfleißes binden und organisieren, kaum eine geringere Bedeutung zu als der produktiven Tätigkeit der erwerbenden Klassen, die jenen Nutzen erst ermöglicht.

Unter den Institutionen, die diesem Zwecke dienen, nimmt den hervorragendsten Platz die Lebensversicherung ein; ihre volkswirtschaftliche Bedeutung geht weit über die bloße Thesaurierungsfunktion der Sparkassen hinaus.

Die Lebensversicherung ist ein Vertrag, durch welchen sich die Versicherungsanstalt verpflichtet, gegen Erhalt jährlicher, nach dem Eintrittsalter des Versicherten sich bemessender Prämien ein Barkapital auszuzahlen, sobald der Versicherte stirbt oder (bei der abgekürzten Versicherung) spätestens beim Erleben eines vorher vereinbarten Zeitpunktes. Der Zweck eines solchen Vertrages ist: D e $\mathbf{n} \mathbf{f} \mathbf{i}$ nanziellen Ertrag der Lebensarbeit des Versicherten seinen Angehörigen auch für den Fall zu sichern, da $\beta$ vorzeitiger Tod es ihm unmöglieh machen sollte, aus seinem Einkom men den Ertrag selbst zu bilden, mit dem er bei unverkïrzter Lebensda u er rechnen d urfte. Die Wirkung des Versicherungsvertrags erhellt am deutlichsten, wenn man sie mit der der Sparkasse vergleicht. Die letztere wirkt auch vermögenbildend; aber sie kumuliert nur die Einlagen, welche wirklich g e m a e ht sind. Die Lebensversicherung dagegen macht die Familie des Versicherten sofort beim Ab-

6) Diese Z. 20, 1101 (1907).

7) Z. f. Industrierecht 1907, 128. 